\title{
Appendix Korinth
}

Die Beschreibung der Stadt geht von 2,1,1 bis 2,5,5. Die gesamte Darstellung Korinths ist von drei Tendenzen bestimmt: 1. Korinth in Griechenland den zweiten Rang hinter Athen einzuräumen (nicht nur geographisch), 2. dezidiert das römische Gepräge Korinths zu betonen, und zwar als etwas positives Neues, welchem später die Ruinen anderer Orte (Sikyon) und die Verkommenheit ihrer Bewohner (besonders der Argiver) gegenübergestellt werden und 3. über die Beschreibung von Religion eine positive Synthese zwischen griechischen und römischen Substraten herzustellen.

Pausanias leitet die Beschreibung ein $(2,1,1)$ mit der Diskreditierung der alt-korinthischen genealogischen Überlieferung (Korinthos sei Sohn des Zeus) des aus dem Geschlecht der Bakchiden (= Dorer) stammenden Korinthers Eumelos (8./7. Jh. v. Chr.); berichtet respektive selektiert werden aus dessen korinthischer Schrift ferner die Motive der Flucht von Korinthern aus dem Gebiet der Stadt (Marathon vor seinem eigenen Vater) und das der Gewalttätigkeit und Ungesetzlichkeit der Dorer (hier: König Epopeus). In Abschnitt 2 kommt Pausanias unmittelbar darauf zu sprechen, dass Korinth nun von römischen Kolonisten bewohnt wird und schließt daran die Erklärung, wie es historisch dazu gekommen ist, wobei die Schuld eindeutig dem Achaiischen Bund zugewiesen wird, d.h. es wird zudem ein in den folgenden Büchern weiter entfaltetes Motiv (mit dem Gipfelpunkt in Buch 7) aufgenommen. Als Konsequenz oder konsequente Bestrafung für den, nach Pausanias ,,vom Strategen des Achaiischen Bundes angezettelten Krieg“" erscheint die Zerstörung Korinths durch den römischen Feldherren Mummius, das von Caesar dann jedoch wieder aufgebaut wird und so der hier im übrigen in keiner Weise dramatisch ausgemalte Schaden wieder gut gemacht scheint. In den Abschnitten 3 und 4 ist die Gegend des Kromyon Hintergrund der Erwähnung des Altar des Melikertes und der Einrichtung der Isthmischen Spiele durch Sisyphos (zugleich Vorverweis auf den folgenden Logos) sowie Schauplatz weiterer Heldentaten des Theseus (Tötung der Sau und Bestrafung und Hinrichtung des Räubers Sinis), was als Verknüpfung mit dem Glanz des athenischen Helden und der mytho-historischen Zeit der Griechen anzusehen ist.

Abschnitt 5: Dass der Isthmus von Korinth nicht durchstochen bzw. seine Durchstechung aufgegeben worden ist, vergegenwärtigt nach Pausanias die Allmacht des Schicksals, indem dem Bericht über zwei weitere gescheiterte Versuche der Isthmus-Durchstechung die Bemerkung angeschlossen ist, dass 
man daran sehen könne, wie schwer es für Menschen ist, „Götterwerk gewaltsam zu ändern“ (Scheitern Alexanders bei der Mimashalbinsel - Fall der Makedonen; Verzicht der Knidier wegen eines hier nicht spezifizierten Orakels - Überblendung knidischer Geschichte). Abschnitt 6: Das Thema und Motiv des Athenischen Vorrangs vor anderen griechischen Städten wird anhand des Götterstreites (Athener rühmten Attika zuerst dafür) diesmal gegenüber Korinth aufgegriffen (Poseidon und Helios streiten um die Stadt, der Streit wird durch Briareos geschlichtet). Die Abschnitte 7 bis 9 enthalten die Beschreibung des Stadions der Isthmischen Spiele und des kleineren Tempels, hier besonders der von Herodes Atticus geweihten Statuengruppe, deren Material (Gold und Elfenbein) hervorgehoben ist; die Adelung der neuen bzw. für Pausanias zeitgenössischen Statuen erfolgt durch die Betonung alter und traditioneller Elemente (sc. der Palaimon-Statue): die Nereiden an der Basis sind, ganz gleich wie andere Altäre dieser Gottheiten in Griechenland, sogar auch der Doto und ihr Tempel im syrischen Gabala (nach Homer eine der Nereiden, wie Pausanias hier vermerkt), wo auch der Peplos aufbewahrt wird. ${ }^{1}$ Abschnitt 9 setzt die Beschreibung mit der Basis und weiteren Statuen fort, wodurch das alte (griechisch-mythische) und neue (römisch-griechische) Korinth gleichermaßen repräsentiert ist (Galene, Meer, Pferd, Ino, Bellerophontes, Pegasos).

2,2,1: Die Heiligkeit des Palaimon-Tempels und besonders des sogenannten Adytons wird dadurch betont, dass nach Pausanias dort geschworene Meineide niemals ungesühnt bleiben, die Verbindung mit altehrwürdigen Einrichtungen wird über ein sich in situ befindliches altes Heiligtum hergestellt, den Altar der Kyklopen. (2) Dem ist wiederum vorrömische bzw. dorische Überlieferung Korinths durch Eumelos gegenübergestellt, das angeblich verborgene Grab des Sisyphos. Erwähnt wird sodann die Kontinuität der Isthmischen Spiele während der Vernichtung Korinths, die zwischenzeitlich von Sikyon ausgerichtet wurden, jetzt aber wieder von Korinth veranstaltet werden. (3) Es folgen die kurzen Beschreibungen der Häfen von Korinth, Lechaion und Kenchrai, die topographisch gleich strukturiert sind wie in der Darstellung Athens und den städtischen Logoi vorangestellt sind. Woher die kommentarlos referierte mythische Genealogie des sonst nicht belegten Leches und des Kenchrias stammt, die Söhne des Poseidon und der Peirene sein sollen, ist nicht zu ermitteln; Pausanias greift jedenfalls die Peirene heraus und merkt an, dass sie in den Ehoien Tochter des Oibalos sei - eine, gemessen an den Maßstäben des Pausanias hinsichtlich des Alters einer Überlieferung, jedenfalls bestätigende und positive Verknüpfung. Auf die Peirene-Quelle wird er zudem in Korinth noch

1 Die lapidare Feststellung und unbezweifelte Tatsache, dass der Peplos im syrischen Gabala aufbewahrt wird, kann man m.E. als eines der in die Bücher vereinzelt aber konsequent und gezielt eingestreuten syrischen Statements lesen. 
eigens lobend zu sprechen kommen. Diese Quelle, ebenso wie das in Kenchrai als Naturwunder beschriebene Bad der Helena nehmen Thema und Motiv des Wasserreichtums in Korinth vorweg. (4) Die Erwähnung des Grabmals des Diogenes, des Kraneions (Bellerophontes) und der Tempel der Aphrodite Melainis sind weitere Monumente oder Zeugen griechisch-römisch-korinthischen Hellenentums und damit Zeichen von Kontinuität und führen auf das Grab der Hetäre Laïs: (5) Sie war so schön, dass sich ,noch heute die Korinther um sie streiten": Das heikle Thema Hetären von Korinth ist damit dezent und außerhalb der eigentlich Stadt Korinth abgehandelt, gleichfalls wird wieder ein Moment der Kontinuität zwischen alten und neuen Korinthern herausgestellt.

Abschnitt (6) geht über zu den Sehenswürdigkeiten der Stadt, wobei vorab ausdrücklich der Hinweis erfolgt, dass es sich dabei um vorwiegend Neues handelt; dies sind an erster Stelle die Heiligtümer auf dem Akrokorinth. (7) Der Pentheus-Logos (Frevel gegen Dionysos) zu einem Holzbild - neueren Datums - das aufgrund eines eigens eingeholten Orakels verfertigt wurde, wie die - neuen - Korinther erzählen, kontrastiert alte griechische korinthische ১Untaten gut zu machen. (8) Die dann folgende Aufzählung von Tychetempel, Pantheon, einem Brunnen mit Poseidon und wasserspeiendem Delphin sowie und zahlreiche Statuen griechischer Götter (Apoll Klarios, zwei Bronzestatuen des Hermes, einer mit Tempel, drei Zeusstatuen: eine ohne Beinamen, ein ZeusChthonios, ein Zeus Hypsistos, Aphrodite von einem zeitgenössischen Künstler, des Hermogenes von Kythera) führt das Thema Wasserreichtum weiter, zeigt aber vor allem anhand der Epiklesen der Götter, dass man sich ganz offensichtlich in einer griechischen Stadt befindet.

Diese Aufzählung wird im gleichen Stil fortgesetzt in 2,3,1 von der Straße nach Lechaion zum Markt mit der Nennung einer Bronze-Athena und dem Tempel der Octavia, der ,Schwester des Augustus, der nach Caesar, dem Gründer des jetzigen Korinth, in Rom regierte“ wie Pausanias eher beiläufig vermerkt. (2) Die von Phaëton und Helios getragenen vergoldeten Wagen auf den Propyläen setzen die Schilderung der sgriechischen ‘ Sehenswürdigkeiten fort und lenken den Blick auf den sich in der Nähe des Propylaions befindlichen bronzenen Herakles, von wo Pausanias' Beschreibung dann zum neben dem Herakles befindlichen Eingang zur Quelle Peirene kommt. An die gleichnamige Quelle knüpft eigener Logos an und führt damit ein schon angedeutetes Motiv für das Lob der heutigen Stadt ein, den Wasserreichtum. Abschnitt (3) ist die Fortsetzung des Peirene-Logos: positiv hervorgehoben sind seine Ausstattung sowie das süße Wasser, und als Moment der Kontinuität ist die Bemerkung anzusehen, dass hier das korinthische Erz eingetaucht wird. (4) Die Qualität des Bronze-Hermes mit einem Widder (an der Straße nach Lechaion) wird von Pausanias durch ein dazu passendes Homer-Zitat evoziert sowie einen Verweis auf die Mysterien der Göttermutter (markierte Erzähllücke 
aufgrund von Eusebie). Dann erwähnt Pausanis weitere Statuen, die sowohl für das alte als auch für das neue Korinth stehen (Poseidon, Leukothea, Palaimon). Weiter geht es in Abschnitt 5 mit dem Thema Wasserreichtum, d.h. dem Verweis auf die große Anzahl von Bädern (städtische und von Hadrian gestiftete), ausfuihrlich erwähnt ist das vom Spartaner Eurykles gestiftete. Er gilt als Günstling des Augustus, was Pausanias hier jedoch nicht eigens sagt; auch Brunnen gibt es reichlich bzw. überall in der Stadt, die reichlich fließendes Wasser hat und außerdem noch von Hadrian aus Stymphelos zugeleitetes; als den schönsten Brunnen bezeichnet Pausanias den des Bellerophontes (neuer Brunnen - alter mythischer Heros Korinths).

Der vom Markt nach Sikyon führende Weg nimmt in Abschnitt 6 über einen weiteren Brunnen (der Glauke) nun das Thema Medea auf und veranschaulicht daran, wie alte grausame Mythen und Riten durch die neuen römischen Bewohner unwirksam werden: In den Brunnen soll Glauke sich gestürzt haben, weil sie glaubte, es sei ein Heilmittel gegen das Gift der Medea; dann wird das Odeion genannt und damit die topographische Überleitung zum Grabmal der Medea-Kinder Mermeros und Pheres, die von den Korinthern gesteinigt und getötet wurden. (7) Weil diese Tat gewaltsam und unrechtmäßig war (vgl. 2,1,1), werden nach einem Orakelspruch Jahresopfer für die Kinder eingerichtet und die Statue der Deima aufgestellt. Wie Pausanias dann hervorhebt „existiert diese auch zu unseren Zeiten noch, das Bild einer grauenhaft dargestellten Frau. Seit Korinth aber von den Römern zerstört wurde und die alten Korinther zugrunde gegangen sind, sind jene Opfer nicht mehr in Gebrauch, auch scheren sich die Knaben nicht mehr das Haupt, noch tragen sie schwarze Gewänder". Ab Abschnitt 8 bis 11 wird der Medea-Mythos in Verbindung mit den Motiven Flucht (aus Athen, später Korinth) und dem Tod ihrer Kinder (durch andere und sie selbst) berichtet; thematisch ist dies eine Weiterführung des Anfangsmotivs und Vorwegnahme der gleich folgenden Schilderung der Könige der Korinther.

2,4,1: Eine Repräsentation der Verbindung von Altem und Neuem ist wiederum das Heiligtum der Athena Chalinitis (neues Heiligtum) und die Zähmung des Pegasos durch Athena, den sie Bellerophon übergibt (alter Mythos). In den Abschnitten 2 bis 4 erfolgt die nachgeschobene Königsgenealogie der alten Korinther, ausgehend von der Nennung Bellerophons. „Dass Bellerophon nicht uneingeschränkt als König regierte, sondern unter Proitos und den Argivern stand, das glaube ich und wer auch immer die Gedichte des Homer nicht bloß oberflächlich gelesen hat. Offenbar gehorchten die Korinther auch nach der Auswanderung des Bellerophon nach Lykien den Herrschern in Argos und Mykene, auch stellten sie keine eigenen Anführer im Feldzug gegen Troja, sondern sie nahmen am Feldzug teil, zusammen mit den Mykenern und den anderen, die Agamemnon anführte". Der Sisyphossohn Ornytion hat einen Sohn Phokos, der nach Tithorea übersiedelt. 
Unter der Herrschaft der Thoassöhne wird Korinth von den Dorern angegriffen - ,das Volk der Korinther wurde in einer Schlacht besiegt und vertrie$b_{e n}{ }^{\text {“. }}$. Die Römer waren also gar nicht die ersten, die die Korinther vertrieben, denn Korinth ist schon nach der Eroberung von den Dorern bewohnt und beherrscht worden durch das Königsgeschlecht der Bakchiden. Ihre Herrschaft dauert fünf Generationen an, bis Telestes von Arieus und Perantas aus Hass ermordet wird. Danach ist die Wahl von Prytanen aus der Familie der Bakchiden Regel, bis Kypselos Tyrann wird und sie vertreibt und damit das Motiv der Vertreibung der Herrscher und Bewohner von Korinth fortgeführt ist. Abschnitt 5 knüpft wieder an mit dem Athena-Chalinitis-Heiligtum, von dem jetzt mitgeteilt wird, es liege beim Theater; in dessen Nähe ist eine nacktes Holzbild des Herakles, das Daidalos gemacht haben soll. ${ }^{2}$

Dann geht es in der Art der Beschreibung Athens weiter mit neuen, d.h. zeitgenössischen Heiligtümern: Pausanias' Hinweis darauf, dass der Kapitolinische Zeus auf griechisch Koryphaios heiße, ist wohl eine weitere Angleichung von Römischem und Griechischem, ebenso wie das alte Gymnasion und die Quelle Lerna, die zu otium einladen, denn man kann sich hier im Sommer abkühlen. Von den Götterbildern wird das erlesene Material hervorgehoben. (6) Die Fortsetzung der Verknüpfung von alt und neu wird hier über den Aufstieg zum Akrokorinth durch eine Parenthese realisiert, in der Pausanias in Erinnerung ruft, dass es „der Berggipfel ist, den Briareos dem Helios als Richter gab und den Helios, wie die Korinther sagen, der Aphrodite überließ"; obenauf werden mehrere Heiligtümer der Isis und des Serapis mit den jeweiligen Beinamen der Göttin bzw. des Heiligtums des Gottes genannt. Dies ist in der Periegese eine Besonderheit Korinths, denn entweder hat so viele ägyptische Heiligtümer sonst keine griechische Stadt, auch nicht Athen, oder Pausanias erwähnt dies nicht. Es folgt ein Heiligtum der Ananke und Bia (griechische Namen und Götter), das man jedoch nicht betreten darf. Dadurch ist seine besondere Heiligkeit impliziert. Ein weiteres Heiligtum der Göttermutter (Mater Magna? Meter?) mit marmornem Bild und Thron sowie weitere Heiligtümer, die griechischen Göttinnen gehören, betonen das griechische Gepräge der Religion. Am Ende der Liste wird wieder ein Bogen zu deren griechisch-korinthischem Hintergrund geschlagen (Hera Bounia, Gründung des Bounos und auf dem Akrokorinth und Aphrodite mit den alten bzw. traditionellen Abzeichen: Waffen).

2,5,1-4: Die Quelle hinter dem Aphrodite-Tempel ist Anlass zu einer dezidierten Mythenkritik, und zwar der Überlieferung, dass diese ein Geschenk des Asopos an Sisyphos sei, damit dieser ihm verrate, wo Zeus die Asopostochter Aigina versteckt habe; nach Erhalt des Geschenkes habe Sisyphos es

2 Dessen Werk damit einerseits weiter inventarisiert wird. Ausführlich dazu Kapitel 2.4.4.1. 
ihm gesagt und soll dafür seine Strafe im Hades bekommen haben. Eine Information, die weder bestätigt noch bestritten wird (die oben 2,3,1 genannte Peirene entspringe hier und fließe unterirdisch in die Stadt), ist Überleitung zu einem Logos der äußeren Form nach über Flüsse, die ihre Namen ändern, der inhaltlich sowohl das Motiv der Unglaubwürdigkeit der Sikyonier und Phliasier andeutet als auch - an sich wohl prominente - griechische und ägyptische Überlieferungen marginalisiert Nach dem Bericht (Abschnitt 2 bis 3), dass der Asopos im Gebiet von Phlious entspringt und dann durch das von Sikyon fließt, um dann im Meer zu münden, exponiert Pausanias die umstrittene Behauptung der Phliasier, dass zwei Inseln und die Stadt Theben (explizit gegen thebanische Überlieferung) nach den Töchtern des Asopos umbenannt bzw. benannt seien (Korkyra, Aigina, Thebe). Der Aussage der Sikyonier und Phliasier, der Asopos werde in Wirklichkeit vom Maiandros gespeist bzw. sei dieser letztlich, werden ähnlich unglaubwürdige Behauptungen der Delier über den Inopos (dieser sei der Nil) und Ägypter (der Nil sei der Euphrat) angeschlossen.

(4) Der folgende Bericht (Weg Akrokorinth - Berge), die Teneaten behaupteten, Trojaner zu sein (von Griechen in Tenedos im Trojanischen Krieg gefangen), ist singulär bei Pausanias. Tenea galt ansonsten im Altertum als geradezu sprichwörtlich glücklich; ferner wird erwähnt, dass es mit Argos an der Zerstörung Mykenes beteiligt war (Strabo 8,6,19; Cicero, Atticus 6,2,3). Da Tenedos bzw. tenedisch seinerseits sprichwörtlich für Härte, Rohheit und Falschheit war, wirkt diese Konstruktion so oder so fiktiv. (5) Der Weg nach Sikyon von Korinth führt prononciert auf das Thema Ruinen als Kriegsfolge; anknüpfend an einen am Weg liegenden verbrannten Tempel stellt Pausanias fest, dass ,auch sonst Kriege um Korinth“ stattfanden, in denen Häuser und Heiligtümer zerstört worden seien. Bezüglich des genannten verbrannten Tempels gibt Pausanias zwei Varianten: es habe sich um einen von Pyrrhos verbrannten Apolltempel gehandelt oder es sei ein durch Feuer vernichteter Zeustempel der Korinther gewesen. 